\title{
Avaliação das principais causas de morbidade hospitalar na região metropolitana de Belém - PA no período de 2014 a 2018
}

\author{
Evaluation of the main causes of hospital morbidity in metropolitan region of Belém - PA in \\ the period from 2014 to 2018
}

Evaluación de las principales causas de morbidad hospitalaria em la región metropolitana de Belém - PA em el período de 2014 a 2018

Karen Giovana Leal Matos ${ }^{1 \star}$, Iris Malato Correa1.

\begin{abstract}
RESUMO
Objetivo: Caracterizar o perfil das internações hospitalares na Região Metropolitana de Belém - PA no período de 2014 a 2018 e avaliar as principais causas de morbidades. Métodos: Estudo transversal, descritivo e quantitativo. Os dados coletados foram realizados com 861.878 pacientes internados, com a relação de internações segundo o cid-10, fatores epidemiológicos e caráter de atendimento. Todos os dados foram obtidos da plataforma DataSUS. Resultados: A maioria pertence ao sexo feminino (58,5\%), com idade entre 20 e 29 anos (22,5\%). O caráter de atendimento prevalente foi o de urgência/emergência ( $85 \%$ ) e o maior motivo de internação relaciona-se à gravidez, parto e puerpério $(24,9 \%)$. Observou-se também subnotificação em relação ao regime de internação, se público ou privado. Conclusão: Na região metropolitana de Belém as mulheres jovens são o maior número de internações, fato que se dá principalmente pelo alto número de internações relacionadas à gravidez, parto e puerpério, caracterizando, assim, esse perfil de internações.
\end{abstract}

Palavras-chave: Hospitalização, Epidemiologia, Perfil de saúde.

\begin{abstract}
Objective: To characterize the profile of hospital admissions in the Metropolitan Region of Belém - PA from 2014 to 2018 and to evaluate the main causes of morbidities. Methods: Cross-sectional, descriptive and quantitative study. The collected data were carried out with 861,878 hospitalized patients, with the hospitalization ratio according to the ICD-10, epidemiological factors and character of care. All data were obtained from the DataSUS platform. Results: Most of them are female (58.5\%), aged between 20 and 29 years old $(22.5 \%)$. The prevalent type of care was urgent / emergency $(85 \%)$ and the biggest reason for hospitalization is related to pregnancy, childbirth and the puerperium (24.9\%). There was also underreporting in relation to the hospitalization regime, whether public or private. Conclusion: In the metropolitan region of Belém, young women are the largest number of hospitalizations, a fact that is mainly due to the high number of hospitalizations related to pregnancy, childbirth and the puerperium, thus characterizing this profile of hospitalizations.
\end{abstract}

Key words: Hospitalization, Epidemiology, Health profile.

\section{RESUMEN}

Objetivo: Caracterizar el perfil de ingresos hospitalarios en la Región Metropolitana de Belém - PA de 2014 a 2018 y evaluar las principales causas de morbilidad. Métodos: Estudio transversal, descriptivo y cuantitativo. Los datos recolectados se realizaron con 861.878 pacientes hospitalizados, con el índice de

${ }^{1}$ Santa Casa de Misericórdia do Pará, Belém - PA. *E-mail: karenlealmatos@gmail.com 
hospitalización según la CIE-10, factores epidemiológicos y carácter de la atención. Todos los datos se obtuvieron de la plataforma DataSUS. Resultados: La mayoría son mujeres $(58,5 \%)$, con edades comprendidas entre 20 y 29 años (22,5\%). El carácter de servicio predominante fue urgente / emergencia (85\%) y el mayor motivo de hospitalización está relacionado con el embarazo, parto y puerperio (24,9\%). También hubo subregistro en relación con el régimen de hospitalización, ya sea público o privado. Conclusión: En la región metropolitana de Belém, las mujeres jóvenes son el mayor número de hospitalizaciones, hecho que se debe principalmente al alto número de hospitalizaciones relacionadas con el embarazo, parto y puerperio, caracterizando así este perfil de hospitalizaciones.

Palabras clave: Hospitalización, Epidemiología, Perfil de salud.

\section{INTRODUÇÃO}

As causas das internações hospitalares no Brasil são um vasto campo de pesquisa. Ao avaliar a internação hospitalar de um país ou cidade, se faz necessário ter conhecimento a respeito do envelhecimento da população, já que os dados de internação estão diretamente relacionados a mudança demográfica e epidemiológico do país. Associado a isso, a redução na taxa de fecundidade devido às mudanças na sociedade e nas próprias estruturas familiares, contribui ainda mais para a modificação das causas de internação (BARBOSA TC, et al., 2019).

Além disso, o aumento da expectativa de vida dos brasileiros, resultante de melhores condições de vida e de saúde, juntamente com a diminuição da mortalidade, também contribuíram para o processo de modificação de internação hospitalar (BARBOSA TC, et al., 2019).

No decorrer dos anos, mudanças no perfil das internações hospitalares no Brasil ocorreram. Até 1980 o Brasil vivenciou o padrão de internação hospitalar com prevalência em doenças infectocontagiosas, dando ênfase para a malária, tuberculose e Síndrome da Imunodeficiência Adquirida (SIDA). Com a endemia de doenças como a malária na maior parte do país e diversos programas nacionais de saúde entrando em vigor, como o Programa Nacional de Imunização (PNI), a lei orgânica de saúde e a implantação do Sistema Único de Saúde (SUS) começou a preconizar políticas de caráter preventivo e curativo, dando início a modificação das causas de internação no país (GOMES HG, et al., 2018).

A partir de então, houve a diminuição de doenças infecciosas, dando espaço para doenças crônicas de caráter progressivo, como as doenças cardiovasculares, neurodegenerativas, dentre outras, representando, portanto, a atual transição epidemiológica vivida pelo Brasil (MARTINS AA, 2018). Para o estudo de conjunto de causas capazes de produzir uma doença e gerar os diagnósticos hospitalares, a Classificação Internacional de Doenças (CID) é utilizada e aplicada há décadas para que os pesquisadores, independentemente da nacionalidade, possam discutir e verificar a prevalência de cauas que geram internação ou não (GOMES HG, et al., 2018).

A morbidade hospitalar foi implantada ao CID-10 pelo Ministério da Saúde através da portaria n. 1.311 de 12 de setembro de 1997, concomitantemente à implementação do Sistema de Informações Hospitalares do Sistema Único de Saúde (SIH/SUS). Essa implementação teve como proposta principal o registro de todos os casos atendidos em hospitais públicos provenientes de internações hospitalares. Desta forma, foi dado início à uma base de dados que reúne, em abrangência nacional, os casos de internações pelo SUS (D’ARTIBALE EF, et al., 2018).

A internação hospitalar pode ser o primeiro passo do caminho que leva à institucionalização e à perda da independência funcional dos pacientes, uma vez que quando ocorre a internação, muitos passam por um período de níveis de mobilidade e atividades reduzidos, o que leva a comprometimentos do estado físico e de vitalidade. Em vista disso, conhecer as causas de internações hospitalares da população por regiões permite traçar estratégias para prevenção e promoção de saúde direcionada para cada população (BARBOSA TC, et al., 2019). 
Dentre as estatísticas mais prevalentes de internações hospitalares, a principal é a materna-neonatal: esta morbidade e mortalidade materna, neonatal e fetal serve como importante termômetro para o controle da saúde materna e infantil nos hospitais que atendem o SUS. Estudos têm sido realizados a fim de traçar um panorama da realidade de morbidade materna e mortalidade neonatal para que estratégias possam ser planejadas e implementadas para maior atenção a esses casos, como no realizado por Moura BLA, et al. (2018).

Os autores relatam também um problema para avaliar e realizar levantamento desses casos, pois não é permitido indicações de afecções maternas no Sistema de Informações sobre Mortalidade (SIM) e nem pelo Sistema de informações sobre Nascidos Vivos (SINASC), logo, é necessário vincular esses dados ao $\mathrm{SIH} / \mathrm{SUS}$, o que dificulta essas avaliações, visto que é o maior índice de internações no Pará ao que se refere o CID-10 (MOURA BLA, et al., 2018).

Atualmente, a realidade dentre os países expressa uma diferença expressiva entre os padrões de internação hospitalar. Por exemplo, enquanto em países como os Estados Unidos tem-se a incidência e prevalência de doenças do aparelho cardiovascular e respiratório, em contrapartida, em países como a África do Sul, ainda se verifica o predomínio de doenças infectocontagiosas, como a tuberculose (GOMES HG, et al., 2018).

Diante dessa conjuntura, este estudo possui como objetivo caracterizar o perfil das internações hospitalares na Região Metropolitana de Belém - PA no período de 2014 a 2018 e avaliar as principais causas de morbidades. Isso se dará através de um levantamento epidemiológico e, bem como o caráter de atendimento dos pacientes, levando em consideração os sete municípios da Região Metropolitana de Belém do Pará: Ananindeua, Benevides, Castanhal, Santa Bárbara do Pará e Santa Izabel do Pará, além da própria capital Belém.

\section{MÉTODOS}

O presente artigo está de acordo com os preceitos da Declaração de Helsinque e do Código de Nuremberg, respeitando as Normas de Pesquisa Envolvendo Seres Humanos (Res. 466/12) do Conselho Nacional de Saúde.

Os dados coletados foram realizados com 861.878 pacientes internados, foi analisada a situação das internações hospitalares no Estado do Pará, especificamente, na região Metropolitana de Belém nos últimos cinco anos, de 2014 a 2018. Os itens "acesso à informação", "informações de saúde (TABNET), "epidemiológicas e morbidade", "morbidade hospitalar do SUS", "geral-por local de internação, a partir de 2014" foram utilizados para a pesquisa. Foram inclusos no estudo todos os casos registrados nas respectivas cidades: Ananindeua, Belém, Benevides, Castanhal, Santa Bárbara e Santa Izabel, notificados no Sistema de Informação de Agravos de Notificação (SINAN/Web).

Os dados foram extraídos do SINAN/Web, de acordo com as variáveis encontradas. Para isso, foram coletados como dados de perfil: número total de pacientes internados na região metropolitana de Belém, a relação de internações segundo o cid-10, os fatores epidemiológicos: sexo, idade e faixa etária e o caráter de atendimento e o regime de internação.

Após o processamento de dados no SINAN/Web foi feita a análise no software Microsoft Excel 2010. As variáveis quantitativas foram descritas por meio de seu valor absoluto, de distribuição de frequências e da taxa de ocorrência. Foram utilizados dados de domínio público, não sendo necessária a submissão do trabalho ao Comitê de Ética em Pesquisa.

\section{RESULTADOS E DISCUSSÃO}

O estudo avaliou especificamente o número de internações nas cidades Metropolitanas de Belém - PA, encontrando um total de 861.878 internações. No total foram incluídas sete cidades, vinte e um CID-10 e avaliados os quais eram as principais causas de internações nos municípios elencados, de acordo com os registros feitos no DATASUS. Os resultados estão resumidos na Tabela 1. 
Tabela 1 - Causas das internações na Região Metropolitana de Belém - PA, segundo a Classificação Internacional de Doenças (CID-10).

\begin{tabular}{|c|c|c|c|c|c|c|c|}
\hline Cid-10 & Ananindeua & Belém & Benevides & Castanhal & Marituba & $\begin{array}{l}\text { Santa } \\
\text { Izabel }\end{array}$ & $\begin{array}{c}\text { Santa } \\
\text { Bárbara }\end{array}$ \\
\hline Infecciosas/parasitárias & 19257 & 45336 & - & 3683 & 2893 & 3487 & 2172 \\
\hline Neoplasias & 2457 & 31280 & - & 1288 & 386 & 137 & 56 \\
\hline $\begin{array}{l}\text { Sangue/órgãos } \\
\text { hemáticos/autoimune }\end{array}$ & 609 & 1876 & - & 382 & 271 & 103 & 31 \\
\hline $\begin{array}{l}\text { Endócrinas/nutricionais/m } \\
\text { etabólicas }\end{array}$ & 3333 & 5772 & - & 853 & 360 & 271 & 150 \\
\hline $\begin{array}{l}\text { Transtornos mentais e } \\
\text { comportamentais }\end{array}$ & 5 & 12366 & 269 & 4 & - & 1 & 9 \\
\hline Sistema Nervoso & 496 & 4342 & - & 65 & 59 & 11 & 174 \\
\hline Olhos e anexos & 239 & 3803 & - & 2 & 6 & 1 & - \\
\hline Ouvidos/apófise mastoide & 20 & 565 & - & 37 & 93 & - & 9 \\
\hline Aparelho circulatório & 9341 & 38196 & - & 3236 & 458 & 872 & 191 \\
\hline Aparelho respiratório & 20673 & 45863 & - & 7947 & 3501 & 2027 & 895 \\
\hline Aparelho digestivo & 21659 & 44978 & - & 5134 & 1601 & 879 & 768 \\
\hline Pele/tecido subcutâneo & 3241 & 20596 & - & 3373 & 441 & 252 & 230 \\
\hline $\begin{array}{l}\text { Sistema Osteomuscular e } \\
\text { tecido conjuntivo }\end{array}$ & 3373 & 8343 & - & 501 & 377 & 132 & 192 \\
\hline Aparelho Geniturinário & 14076 & 21552 & - & 3906 & 1208 & 1162 & 618 \\
\hline Gravidez, parto/puerpério & 32057 & 142061 & - & 26780 & 11986 & 825 & 1083 \\
\hline Afecções perinatais & 3978 & 18397 & - & 3957 & 1557 & 14 & 4 \\
\hline $\begin{array}{l}\text { Malformações } \\
\text { congênitas/anom. Crom. }\end{array}$ & 89 & 4937 & - & 128 & 111 & - & 1 \\
\hline $\begin{array}{l}\text { Sintomas e sinais/achados } \\
\text { anormais em lab. }\end{array}$ & 4634 & 9140 & - & 540 & 46 & 10 & 258 \\
\hline Lesões eventuais & 45134 & 64182 & - & 11033 & 3498 & 159 & 295 \\
\hline Causas externas & 33 & 37 & - & - & 2 & - & - \\
\hline $\begin{array}{l}\text { Contatos com serviços de } \\
\text { saúde }\end{array}$ & 1636 & 26188 & - & 5071 & 1178 & - & 1 \\
\hline
\end{tabular}

Fonte: Matos KGL e Correa IM, 2021.

As cidades avaliadas e seus respectivos números absolutos e porcentagem de internação foram: Ananindeua (n: 186.340; 21,6\%), Benevides (n:269; 0,03\%), Castanhal (n: 77.920; 9\%), Marituba (n: 30.032; 3,5\%), Santa Bárbara do Pará (n: 7137; 0,8\%) e a capital Belém (n: 549.810; 63,8\%). Observou-se uma prevalência exponencial na capital Belém, com mais da metade do número de internações, devido principalmente ao número de pacientes referenciados aos hospitais e outros setores da capital.

No presente estudo houve a prevalência de internações principalmente devido à gravidez e parto/puerpério, o que alcançou cerca de $24.9 \%$ do total, internação, esta, situada no capítulo XV do CID-10. 
Essa internação configura-se como caráter de emergência, visto que, embora muito comum, não há como prever o momento do seu acontecimento, além do que, quando iniciado, não há como adiá-lo (D'ARTIBALE $E F$, et al., 2018).

Observou-se ainda sobre internações devido gravidez, parto/puerpério a prevalência na cidade de Belém, a capital registrou um total de $66,1 \%$, mostrando dominância, o que já era de se esperar tendo em vista a presença do centro hospitalar: Fundação Santa Casa de Misericórdia do Pará (FSCMP) que recebe diariamente pacientes das mais diversas regiões. O estudo encontra-se consonante com Gomes HG, et al. (2018) e Castro GG, et al. (2018) que dentre as causas das internações, gravidez, parto/puerpério apresentaram 20,93\% e 33,1\%, respectivamente, observado como uma das principais causas.

Essa tendência na predominância de internações ocorre também em outros estados, como em Sergipe, segundo um levantamento feito por Cardoso LCC, et al. (2019), onde apontaram que a gravidez, parto e puerpério representaram 33\% de todas as internações hospitalares entre os anos de 2013 a 2018, ou seja, $1 / 3$ dos casos totais pro estado. Embora a internação na gravidez/parto seja necessária e por ser de caráter emergencial, ainda existem algumas intercorrências que também podem estar relacionados a internação ou a prolongação dela, como Pré-eclâmpsia grave, amniorrexe prematura e oligohidramnio (MEDEIROS FDA, et al., 2020).

Na população infantil, diferentemente da população adulta, há um predomínio de doenças agudas que formam parte da lista brasileira de Internações por Condições Sensíveis à Atenção Primária (ICSAP), portanto são evitáveis com a assistência oportuna dos serviços de Atenção Primária à Saúde (APS). Especialmente sobre as afecções perinatais, e o fato de poderem ser evitadas, elas são responsáveis por grande parcela das internações de crianças menores de um ano e a principal causa do óbito infantil no Brasil (PINTO LF, et al., 2019). No Brasil os gastos do SUS com ICSAP sofreram diminuições gradativas periódicas, como mostrado no estudo realizado entre 2000 e 2013 por Souza DK e Peixoto SV (2017).

Como segunda causa, temos as lesões eventuais, presente, esta, no Capítulo XIX do CID-10. O capítulo engloba lesões, envenenamento e algumas outras consequências de causas externas. Dentre as lesões eventuais destaca-se na região Norte, de forma geral, os traumas ocasionados por acidentes de trânsito: responsáveis por 3.400 óbitos diariamente no mundo, no ano de 2012 (MALTA DC, et al., 2016). A cidade de Belém ficou responsável pela maioria dos casos registrados, 51,6\%, no entanto, o município de Ananindeua que abrange o maior centro de referência do estado: o Hospital Metropolitano de Urgência e Emergência (HMUE). Este fator pode estar relacionado com o maior número de habitantes na capital em comparação com Ananindeua.

No Brasil há também uma elevada taxa de internações em decorrência de lesões por Acidente de Transporte Terrestres (ATT). Só no ano de 2013 foram contabilizados 170.805 casos registrados no SIH/SUS de internações por esta causa, sendo o sexo masculino, predominante, chegando a 134,5 casos para cada 100 mil homens no país, com faixa etária de 20 a 39 anos e tendo motociclistas no topo dessa lista (ANDRADE SSCA E JORGE MHPM, 2017). Só no estado do Rio Grande do Norte entre 2008 e 2016 os acidentes com motocicletas somaram 15.233 internações para o SUS, com gastos atingindo um montante de quase dezoito milhões de reais (AZEVEDO UN, et al., 2017).

Os acidentes de trânsito são mais frequentes entre moradores das regiões Norte, Nordeste e CentroOeste. Em Belém há também números expressivos para esses acidentes, havendo média de 23,4 por dia, sendo eles a sua maioria ocorrendo nas sextas-feiras, entre os horários de 18:00 e 20:59 e a sua maioria ocorrendo apenas danos materiais, não carecendo de internações, porém com $11 \%$ ainda necessitando de atendimento em hospitais (SANTOS AS \& BECHIS IO, 2019).

Como terceira causa encontra-se as afecções respiratórias. As doenças respiratórias, tanto as agudas quanto as crônicas, são importantes causa de morbimortalidade. Gomes HG, et al (2018) concluíram que essa causa é responsável pela morbidade de $19,50 \%$ do seu estudo. No Brasil as internações por doenças respiratórias estão ligadas principalmente à pneumonia, que pode ser causada por diversos fatores como concluiu Ramos D, et al. (2019), onde percebeu que as internações aumentam significativamente por 
pneumonia no período de queima de cana-de-açúcar. Essas internações podem aumentar também devido a fatores como qualidade do ar (LEÃO H, et al., 2018). No presente estudo, houve 80.906 internações devido essa causa.

O estudo de Rosa AM, et al. (2015), no período de seis anos da série histórica, também mostrou o alto índice de internações por doenças respiratórias, representando um total de $36 \%$ dos internados na rede SUS no município de Tangará da Serra. Só pneumonia internou 4 vezes mais o esperado quando avaliado crianças menores de cinco anos. Isso mostra que essas doenças também precisam de atenção especial nas estratégias preventivas.

Outras causas importantes que merecem a atenção pelo alto número de casos são as infecciosas/parasitárias e as do trato digestório. As doenças infecciosas nos países do hemisfério sul, principalmente os com perfil socioeconômico menos favorável ainda são apontados como importantes causadores de internações hospitalares, assim como morbimortalidade. No Brasil, um estudo realizado no estado de Minas Gerais analisou a enfermaria de uma Unidade de Doenças infecto-parasitárias e concluiu que somente em um ano 267 pacientes foram internados por estas causas somente nessa unidade, sendo $70 \%$ do sexo masculino, com idade média de 45 anos (CUNHA LI, et al., 2020). Essas doenças também estão diretamente ligadas com a precariedade do saneamento básico e devido ao Brasil se tratar de um país em desenvolvimento sofre com esse problema, o que ainda gera muitos casos com internações (PIMENTEL JMF, et al., 2020).

Algumas doenças também afetam o trato digestório e isso pode justificar a alta incidência dessas internações no presente estudo. Diarréia e febres entéricas estão relacionadas, mas há outros estudos mostrando outros tipos de doenças gastrointestinais carecendo o paciente de internações, principalmente em crianças e recém-nascidos (WASUM FD, et al., 2019). Esse perfil de internação está diretamente ligado às condições precárias de saneamento e higiene que muitas famílias vivem, principalmente em países subdesenvolvidos ou em desenvolvimento, como o nosso.

Em relação a faixa-etária, os pacientes entre $20-29$ anos prevaleceram, representando $22,5 \%$ do total dentre os municípios da região metropolitana de Belém, sendo a cidade de Belém com a maior parte: representando $59,6 \%$ do total (Tabela 2). Observa-se um predomínio da população economicamente ativa que está mais suscetível as diversas patologias, de causas externas ou internas. Mostrando resultado similar ao de Gomes HG, et al. (2018) e Castro GG, et al. (2018), no qual a faixa etária predominante nas internações foi também de 20 a 29 anos. Os maiores motivos das internações relacionam-se à gravidez, parto e puerpério, o que condiz com a faixa etária predominante.

Tabela 2 - Caracterização das internações segundo faixa-etária na Região Metropolitana de Belém - PA.

\begin{tabular}{cccccccc}
\hline Município & $\begin{array}{c}\text { Menor } \mathbf{1} \\
\text { ano }\end{array}$ & $\begin{array}{c}\mathbf{1} \text { a 4 } \\
\text { anos }\end{array}$ & $\begin{array}{c}\mathbf{5} \text { a } \mathbf{9} \\
\text { anos }\end{array}$ & $\begin{array}{c}\mathbf{1 0} \text { a 14 } \\
\text { anos }\end{array}$ & $\begin{array}{c}\mathbf{1 5} \text { a 19 } \\
\text { anos }\end{array}$ & $\begin{array}{c}\mathbf{2 0} \text { a 29 } \\
\text { anos }\end{array}$ & $\begin{array}{c}\mathbf{3 0} \text { a 39 } \\
\text { anos }\end{array}$ \\
\hline Ananindeua & 7831 & 8467 & 4850 & 4670 & 17112 & 47363 & 33733 \\
\hline Belém & 43223 & 37289 & 23542 & 20889 & 48804 & 115700 & 77248 \\
\hline Benevides & - & - & - & - & - & - & - \\
\hline Castanhal & 6238 & 5107 & 4339 & 3795 & 9192 & 20049 & 10336 \\
\hline Marituba & 2755 & 1996 & 1121 & 1084 & 4532 & 7921 & 3326 \\
\hline Santa Izabel & 418 & 1413 & 662 & 482 & 772 & 1621 & 1265 \\
\hline Santa Bárbara & 128 & 779 & 478 & 332 & 759 & 1438 & 927 \\
\hline
\end{tabular}

Fonte: Matos KGL e Correa IM, 2021.

Os pacientes idosos também são um grupo importante se tratando de internações e no presente estudo houve o total de $14,65 \%$ de pacientes idosos contabilizados. Dando ênfase para as doenças crônico- 
degenerativas e causas oncológicas. As faixas etárias avançadas apresentaram as maiores taxas de internação para doenças respiratórias. Esse crescimento é influenciado, ainda, por diversos fatores, como maior susceptibilidade imunológica dos idosos, presença de maior número de comorbidades nessa faixa etária e exposições prévias diferenciadas aos fatores de risco (ocupacionais, tabagismo e outras) (SANTOS MAS, et al., 2015). Nessa faixa etária é comum internações por doenças do aparelho respiratório, circulatório e também neoplasias (TEIXEIRA JJM, et al., 2017), bem como, em menor escala, agressões físicas (CASTRO VC, et al., 2018).

Os problemas de saúde relacionados ao envelhecimento estão ligados a diversos fatores em geral, esse padrão é observado tanto em países desenvolvidos como nos em desenvolvimento. Naturalmente isso faz com que idosos consumam mais o sistema de saúde e permaneçam por mais tempo. Esse alta procura de idosos para internações hospitalares também foi notada em outro estudo realizado por Amaral ACS, et al (2004), observaram também que os idosos representavam quase $30 \%$ do total de internações dos hospitais universitários, sendo os demais hospitais essa taxa ainda maior, permeando entre causas mais simples, como catarata senil, até casos mais graves, como insuficiência cardíaca ou bloqueio atrioventricular.

Avaliou-se ainda o caráter de atendimento (eletivo, urgência, outros tipos de acidentes de trabalho e outros tipos de lesões e envenenamento por agentes químico físicos) e regime de internação (público ou privado) (Tabelas 3 e 4). Quanto ao caráter de atendimento predominou o caráter de urgência ( $\mathrm{n}$ : 653.345 ; 85\%), seguido do caráter eletivo, com um total de 114.894 internações. Estudo de Gomes HG, et al. (2018) tiveram em seu estudo a predominância do caráter de atendimento por urgência.

Tabela 3 - Internações na Região Metropolitana de Belém - PA, segundo o caráter de atendimento.

\begin{tabular}{ccccc}
\hline Município & Eletivo & Urgência & Outros ac trab. & Outras caus. ext. \\
\hline Ananindeua & 13732 & 172606 & 2 & - \\
\hline Belém & 101051 & 448757 & 1 & 1 \\
\hline Benevides & 2 & 2230 & - & - \\
\hline Castanhal & 1 & 24060 & - & 1 \\
\hline Marituba & 99 & 445 & - & - \\
\hline Santa Izabel & 4 & 2155 & - & - \\
\hline Santa Bárbara & 5 & 3092 & - &
\end{tabular}

Fonte: Matos KGL e Correa IM, 2021.

Tabela 4 - Internações na Região Metropolitana de Belém - PA segundo o regimento.

\begin{tabular}{ccccc}
\hline Município & Público & Privado & Ignorado & Total \\
\hline Ananindeua & 19968 & 48022 & 118350 & 186340 \\
\hline Belém & 99278 & 93299 & 357233 & 549810 \\
\hline Benevides & 242 & - & 27 & 269 \\
\hline Castanhal & 5186 & 27202 & 45532 & 77920 \\
\hline Marituba & 1818 & 10120 & 18094 & 30032 \\
\hline Santa Izabel & - & 9390 & 980 & 10370 \\
\hline Santa Bárbara & - & 1515 & 5622 & 7137 \\
\hline
\end{tabular}

Fonte: Matos KGL e Correa IM, 2021. 
Considerando o sexo dos pacientes internados, houve a prevalência do sexo feminino ( $\mathrm{n}: 458334 ; 58,5 \%)$, já o sexo masculino representou $41,5 \%$. Importante ressaltar a subnotificação em relação a essa variável, na qual 7.792 pacientes não foram categorizando nessa constante (Tabela 5). Gomes HG, et al. (2018) conclui em seu estudo que a maioria das internações hospitalares foi de pacientes do sexo feminino. Nascimento $A B$ (2015) demonstra em seu estudo o número de internações e período de permanência do paciente, concluindo que crianças, adultos e idosos do sexo feminino tiveram mediana de tempo de internação hospitalar maior em comparação aos demais grupos etários, por sexo.

A prevalência do perfil de pacientes internadas ser sexo feminino se deve provavelmente pelo alto número de internações por conta de gravidez/parto/puerpério, pois outros estudos que avaliam internações somente por causas externas (que elimina esses fatores relacionados à gravidez) concluiu que $71,8 \%$ dessas internações foram da população masculina, tendo essa predominância em todos os grupos (agressões, quedas, acidentes de trânsito, de ciclistas ou motociclistas) (SOUZA CNS, et al., 2018).

Tabela 5 - Caracterização das internações segundo sexo na Região Metropolitana de Belém - PA.

\begin{tabular}{cccc}
\hline Município & Masculino & Feminino & Total \\
\hline Ananindeua & 82133 & 104207 & 186340 \\
\hline Belém & 226513 & 323297 & 549810 \\
\hline Benevides & 135 & 134 & 269 \\
\hline Marituba & 9710 & 20322 & 30032 \\
\hline Santa Izabel & 4365 & 6005 & 10370 \\
\hline Santa Bárbara & 2768 & 4369 & 7137 \\
\hline
\end{tabular}

Fonte: Matos KGL e Correa IM, 2021.

Os maiores motivos das internações tanto no presente estudo quanto no de Gomes HG, et al. (2018) relacionam-se à gravidez, parto e puerpério, estão consonantes os resultados de faixa etária e gênero sexual predominante, inclusive com o próprio caráter de urgência/emergência majoritário. Importante citar ainda, a subnotificação relacionada a esse dado estatístico, tendo 93.634 pacientes não inclusos na plataforma DATASUS.

Quanto ao regime de internação, observou-se a prevalência de casos ignorados (n: $545.838 ; 63 \%$ ) mostrando a necessidade de se obter dados fidedignos em relação a essa variável, e mostrando ainda a falta de dados para constar em presente e futuras pesquisas. Segue-se o predomínio do regime privado (n: 189.548) e público (n: 126.492). Sendo um dado irrelevante para pesquisa devido a quantidade de subnotificação, comprometendo seu valor.

\section{CONSIDERAÇÕES FINAIS}

Nesse trabalho pudemos observar que a maioria das internações hospitalares são do sexo feminino com faixa etária entre 20 a 29 anos, sendo a urgência predominante. Esse fato se deve provavelmente as maiorias das internações relacionarem-se à gravidez, parto e puerpério, o que condiz com a faixa etária e gênero predominante, inclusive com o próprio caráter de urgência/emergência. Respectivamente, em segundo e terceiro lugar, destacam-se as lesões eventuais e doenças do aparelho respiratório. Com isso, dar a devida importância às principais causas de internações com trabalho eficiente da equipe de saúde torna-se resolutiva, sobretudo quando o pensamento envolve a atenção básica.

\section{REFERÊNCIAS}

1. AMARAL ACS, et al. Perfil de morbidade e de mortalidade de pacientes idosos hospitalizados. Cadernos de Saúde Pública, 2004; 20: 1617-1626. 
2. ANDRADE SSCA, JORGE MHPM. Internações hospitalares por lesões decorrentes de acidente de transporte terrestre no Brasil. Epidemiologia e Serviços de Saúde, 2017; 26: 31-38.

3. AZEVEDO UN, et al. Internações por acidentes de transporte terrestre envolvendo motocicletas. Revista Brasileira em Promoção da Saúde, 2017; 4: 1-10.

4. BARBOSA T, et al. Causas de internações hospitalares em idosos por regiões do Brasil. Revista de Saúde Pública do Paraná, 2019; 2: 70-81.

5. CARDOSO LCC, et al. Caracterização do perfil das internações para gravidez, parto e puerpério entre os anos de 2013 e 2018 no estado de Sergipe. Congresso Internacional de Enfermagem, 2019; 1: 1-5.

6. CASTRO GG, et al. Perfil das interações hospitalares em município de Minas Gerais. Revista Família, Cicloes de vida e Saúde no Contexto Social, 2018; 6: 45-52.

7. CASTRO VC, et al. Violência contra os idosos brasileiros: uma análise das internações hospitalares. Revita Brasileira de Enfermagem, 2018; 71: 777-785.

8. CUNHA LI, et al. Perfil epidemiológico e clínico das internações da Enfermaria da Unidade de Doenças Infecto Parasitárias do Hospital de Clínicas da Universidade Federal do Triângulo Mineiro. Brazilian Journal of Health Review, 2020; 3: 16779-16788.

9. D'ARTIBALE E, et al. Morbimortalidade dos pacientes adultos internadosem unidade aberta. Estudo epidemiológicoHospital Santa Rosa - Cuiabá (MT). Revista Científica do Hospital Santa Rosa, 2018; 1: 64-74.

10. GOMES HG, et al. Perfil das internações hospitalares no Brasil no período de 2013 a 2017. Revista Interdisciplinar, 2018; 10: 96-104.

11. LEÃO H, et al. A qualidade do ar influencia as internações hospitalares por doenças respiratórias em crianças? Uma revisão sistemática. Cardiorespiratory, Physiotherapy, Critical Care and Rehabilitation, 2019; 9: 55-70.

12. MALTA DC, et al. Lesões no trânsito e uso de equipamento de proteção na população brasileira, segundo estudo de base populacional. Ciência \& Saúde Coletiva, 2016; 21: 399-410.

13. MARTINS AA. Estudo da morbidade hospitalar do SUS pela Análise de Componentes Principais. Trabalho de Conclusão de Curso (Estatística) - Universidade Federal de Uberlândia, Uberlândia, 2018; 138 p.

14. MEDEIROS FDA, et al. Aspectos relacionados às internações por intercorrências gestacionais. Enfermagem em foco (Brasília), 2020; 1:41-48.

15. MOURA BLA, et al. Internações por complicações obstétricas na gestação e desfechos maternos e perinatais, em uma coorte de gestantes no Sistema Únicode Saúde no Município de São Paulo, Brasil. Cadernos de Saúde Pública, 2018; 34: 1-13.

16. NASCIMENTO AB. Gerenciamento de leitos hospitalares: análise conjunta do tempo de internação com indicadores demográficos e epidemiológicos. Revista de Enfermagem e Atenção A Saúde, 2015; 4: 65-78.

17. PIMENTEL JMF, et al. Internações hospitalares por doenças relacionadas ao saneamento básico inadequeado na Bahia, de 2010 a 2016. Brazilian Journal of Health Review, 2020; 3: 7945-7957.

18. PINTO LF, et al. Internações por Condições Sensíveis à Atenção Primária (ICSAP) no Distrito Federal: comparação com outras capitais brasileiras no período de 2009 a 2018. Ciência \& Saúde Coletiva, 2019; 24: 2105-2114.

19. RAMOS D, et al. Impacto da queima de cana-de-açúcar sobre internações hospitalares por doenças respiratórias. Ciência \& Saúde Coletiva, 2019; 24: 4133-4140.

20. ROSA AM, et al. Análise das internações por doenças respiratórias em Tangaráda Serra - Amazônia Brasileira. Jornal Brasileiro de Pneumologia, 2008; 34: 575- 582.

21. SANTOS AS, BECHIS IO. Caracterização, mapeamento e análise dos acidentes de trânsito do município de Belém. Trabalho de Conclusão de Curso (Engenharia Civil) - Instituto de Tecnologia. Universidade Federal do Pará, Belém, 2016; $237 p$.

22. SANTOS MAS, et al. Tendências da morbidade hospitalar por doenças crônicas não transmissíveis no Brasil, 2002 a 2012. Epidemiologia e Serviços de Saúde, 2015; 24: 389-398.

23. SOUZA CNS, et al. Diferenciais de sexo nas internações referentes a importantes causas externas no estado de Sergipe em 2017. Congresso Nacional de Enfermagem, 2018; 1: 1-5.

24. SOUZA DK, PEIXOTO SV. Estudo descritivo da evolução dos gastos com internações hospitalares por condições sensíveis à atenção primária no Brasil, 2000-2013. Epidemiologia e Serviços de Saúde, 2017; 26: 285-294.

25. TEIXEIRA JJM, et al. Perfil de internações de idosos. Revista da Sociedade Brasileira de Clínica Médica, 2017; 1: 15-20.

26. WASUM FD, et al. Prevalência de itnernações hospitalares por diarreia e gastroenterite em menores de um ano. Brazilian Journal of Health Revieiw, 2019; 2: 99-105. 